\title{
Attenuation of Osteoarthritis Progression via a Combination of Intra-articular Injection of Synovial Membrane-derived MSCs (SMMSCs), Platelet Rich Plasma (PRP) and Conditioned Medium (Secretome)
}

\section{Sara Sadat Nabavizadeh}

Shiraz University of Medical Sciences

Tahereh Talaei-Khozani

Shiraz University of Medical Sciences

Moein Zarei

West Pomeranian University of Technology: Zachodniopomorski Uniwersytet Technologiczny w

Szczecinie

\section{Shahrokh Zare}

Shiraz University of Medical Sciences Medical School

\section{Omid Koohi Hosseinabadi}

Shiraz University of Medical Sciences Medical School

Nader Tanideh

Shiraz University of Medical Sciences Medical School

Sajad Daneshi ( $\sim$ sa.daneshi@yahoo.com )

Shiraz University of Medical Sciences https://orcid.org/0000-0002-3804-1444

\section{Research}

Keywords: Osteoarthritis, Mesenchymal stem cells, PRP, Secretome, Rat

Posted Date: July 13th, 2021

DOl: https://doi.org/10.21203/rs.3.rs-680028/v1

License: (c) (1) This work is licensed under a Creative Commons Attribution 4.0 International License. Read Full License 


\section{Abstract}

Introduction: Osteoarthritis $(\mathrm{OA})$ as a progressive destructive disease of articular cartilage is the most common joint disease characterized by reduction of joint cartilage thickness, demolition of cartilage surface and new bone formation. To overcome these problems, the purpose of the current research was to evaluate and compare the in vivo effects of Synovial membrane-derived MSCs (SMMSCs), platelet rich plasma (PRP) and conditioned medium (secretome) on collagenase II-induced rat knee osteoarthritis (KOA) remedy.

Methods: For the first step, SMMSCs were isolated and characterized. Also, secretome was collected from SMMSCs culture. Furthermore, PRP was collect from the rat heart venous blood. Second, two injection of collagenase II with an interval of 3 days was performed in the knee intra- articular space to induce osteoarthritis. Two weeks later, animals were randomly divided into 6 groups. Control group without treatment, positive group: taken an intra-articular Hyalgan injection $(0.1 \mathrm{cc})$, treatment groups taken an intra-articular injection of; treatment 1: SMMSCs $(5 \times 106)$, treatment 2: SMMSCs $(5 \times 106)+$ secretome $(50 \mu \mathrm{l})$, treatment 3: SMMSCs $(5 \times 106)+$ PRP $(50 \mu \mathrm{l})$, and treatment 4: SMMSCs $(5 \times 106)+$ secretome $(50 \mu l)+\operatorname{PRP}(50 \mu l)$. Three months later, rats were sacrificed and the following assessments were executed: radiography, histopathology, and immunohistochemistry.

Results: Our findings represented that a combination of the SMMSCs with PRP and secretome had a considerable effect on GAGs and collagen II contents, articular cartilage preservation, compared with other groups. In addition, combination of the SMMSCs with PRP and secretome showed the lowest expression of mmp3, while SOX9 had the highest expression in comparision with other groups. Also, SMMSCs -injected groups demonstrated better results compared with positive and control groups.

Conclusions: Injection a combination of the SMMSCs with PRP and secretome resulted in better efficacy in terms of joint space width, articular cartilage surface continuity and integrity, sub chondral bone and ECM constituents such as collagen II. Indeed, transplantation of this combination could be considered as a promising therapy for patients with KOA.

\section{Introduction}

Osteoarthritis (OA) as a polygenic disease is a debilitating, irreversible, degenerative, and severe joints disorder like in the hip, knee, and hand in humans. OA associates with articular cartilage demolition and decrement, hypertrophy of the synovium, alterations in the ligaments, capsule and synovial membrane, osteophytes formation or aberrant bone outgrowths, and increased thickness of the subchondral bone in which leads to alleviate daily living activity [1]. Universally, over 250 million patients are suffered from the OA especially knee osteoarthritis (KOA) whereby $30 \%$ of older people aged more than 60 years have gait dysfunction, pain, instability, stiffness, and their joint space is declined [2,3]. To date, different methods and materials have been applied in order to cure for KOA. In recent years, an increasing number of experiments have shown that current procedures such as exercises, medications, physical therapy, and 
surgery have not been fully successful in treatment of KOA and prevention of damage to the joint tissue [4]. Furthermore, knee joint replacement as a gold procedure to cure KOA is painful and expensive along with inflammation and bleeding. Taking all these problems and limitations into consideration, an outlook of therapy on future has been focused on stem cells and biological products [5].

A multitude of studies have applied mesenchymal stem cells (MSCs) as a potent tool to cure different diseases. Their characteristics like self renewably, anti-inflammatory and immunomodulatory effects have made them as a promising option for treatment of diseases [6]. Moreover, MSCs, multipotent stromal cells, can differentiate into multiple lineages of cells including chondrocytes (cartilage) and osteoblasts (bone) whereby limitations of poor intrinsic regeneration of cartilage tissue lesion due to the disability of resident articular chondrocytes to secrete a matrix could be reparated [7]. Derived-MSCs of various sources such as amniotic fluid, bone marrow, umbilical cord, adipose tissue, synovial fluid, have been evaluated in a lot of experimental studies about different diseases. In addition to general properties of MSCs, their paracrine activity, angiogenesis and chondrogenic potential made stem cell therapy an interested subject for treatment of KOA wherein extracellular matrix is damaged and chondrocyte function decreased [8].

Experimental Findings have shown that post intra-articular injection of MSCs, their proliferation decreased and differentiation into cartilage cells impaired. Nonetheless, it is notable that conditioned medium of MSCs known as secretome or MSCs paracrine is containing hormones, signaling biomolecules, chemokines, extracellular vesicles, growth factors, and cytokines affecting host cells functions such as differentiation, immigration, and secretion. Besides, secretome have simply freezing which help it to be conserved for a long time $[9,10]$.

Platelet-rich plasma (PRP) as an autologous blood derived product without transfer of blood diseases and immunological reactions is safe [11]. Also regarding the treatment of KOA, It is reported that PRP has similar effects like intra-articular injection of hyaluronic acid. In addition, PRP, a volume of plasma with a platelet concentration ( 3 to 5 fold ), contains growth factors such as platelet-derived growth factor (PDGF) and transforming growth factor (TGF- $\beta$ ), which in turn can stimulate responsible cells to repair and regenerate destructed cartilage and bone tissues in KOA patients which make it promising option. PRP has also been revealed to reproduce hyaluronan synthesis, enhance angiogenesis, and moderate inflammation [12].

Hence, to achieve the desired design targets, we have attempted to comparatively survey the effectiveness of the Synovial membrane-derived MSCs, PRP and secretome in KOA betterment.

\section{Materials And Methods}

\section{Isolation and culture of rat synovial membrane-derived MSCs (SMMSCs)}


The current research was performed at Center of Comparative and Experimental Medicine, Shiraz University of Medical Sciences, Shiraz, Iran. Adult male Sprague Dawley rats were sacrificed in a CO2 chamber. Under sterile conditions, the knee joint was exposed and synovial membrane samples were harvested from the inner side of the articular capsule. Synovial membrane was washed using sterile phosphate buffered saline (PBS; Gibco, USA) to eliminate unwanted tissues and thereupon minced into small pieces and transferred in a tissue plate. Then, collagenase type 1 (Sigma-Aldrich, USA) was added to digest the explanted tissues at $37^{\circ} \mathrm{C}$. One hour later, a $70-\mu \mathrm{m}$ nylon filter was used to filter the digested cells to yield single-cell suspensions. Thereafter, isolated cells were cultured in a growth medium containing Dulbecco's modified Eagle's medium (DMEM) low glucose, 10\% fetal bovine serum (FBS; Gibco, USA) and $100 \mathrm{U} / \mathrm{mL}$ penicillin-streptomycin (Gibco, USA) and incubated in 5\% CO2 at a temperature of $37^{\circ} \mathrm{C}$. Post $48 \mathrm{hr}$, the medium was gently removed and fresh medium was added until at $80 \%$ confluency. Next, Trypsin-EDTA (Gibco, USA) was used to detach the cells, which in turn transferred to new flasks. Cell culture was continued up to passage 3 for characterization and intra-articular injection.

\section{Phenotypic characterization}

The International Society of Cell Therapy was applied to characterize the SMMSCs. Subsequently, flow cytometry (BD FACSCalibur, flow cytometer, USA) was implemented to evaluate surface marker expression (CD34, CD44, CD45, and CD90) of SMMSCs. The data analysis was performed with Flow Jo software (version 10.1).

Furthermore, multi-potentiality was assessed by in vitro adipogenic and osteogenic differentiation.

\section{Adipogenesis}

SMMSCs at passage 3 were incubated in adipogenic media to differentiate into osteoblasts for 2 weeks. In addition, a control media containing SMMSCs at passage 3 was preserved along with osteogenic media. The control medium consisted of DMEM (Gibco, USA), supplemented with 10\% FBS (Gibco, USA) and 1\%penicillin/streptomycin (Gibco, USA). The adipogenic medium contained DMEM, 15\% FBS, 100 $\mu \mathrm{M}$ L-ascorbic acid, $200 \mu \mathrm{M}$ Indomethacin, $1000 \mathrm{nM}$ insulin (Sigma-Aldrich), and $100 \mathrm{nM}$ dexamethasone (Sigma-Aldrich). The medium was substituted 2 times a week. Finally, the cultured cells were fixed in $4 \%$ paraformaldehyde (PFA) for 20 min. Next, Oil Red-O Solution was applied to stain lipid vacuoles for 20 min at room temperature.

\section{Osteogenesis}

SMMSCs at passage 3 were incubated in osteogenic media to differentiate into osteoblasts for 2 weeks. In addition, a control media containing SMMSCs at passage 3 was preserved along with osteogenic media. The control medium contained DMEM (Gibco, USA), 10\% FBS (Gibco, USA) and 1\% penicillin/streptomycin (Gibco, USA). The osteogenic medium consist of DMEM, 15\% FBS (Gibco, USA), $100 \mu \mathrm{M} \mathrm{L-ascorbic} \mathrm{acid,} 10 \mathrm{mM}$ glycerol 3- phosphate, and $100 \mathrm{nM}$ dexamethasone (Sigma-Aldrich). The medium was changed 2 times a week. Eventually, SMMSCs were fixed in paraformaldehyde (4\%) for 20 min and then stained with Alizarin Red solution for 20 min at room temperature to stain calcium deposits. 


\section{Conditioned medium (secretome) preparation}

Tissue-culture dishes $(150 \mathrm{~mm})$ were used to SMMSCs culture at $37^{\circ} \mathrm{C}$ with $10 \% \mathrm{CO} 2$ and an atmosphere of $90 \%$ air. As the cell culture in DEMD became confluent, PBS was applied to rinse SMMSCs and the growth medium was substituted with DMEM. After 24 hours, the conditioned medium was collected and centrifuged twice, first at $500 \times \mathrm{g}$ for $10 \mathrm{~min}$ and afterward at 3,000 $\times \mathrm{g}$ for $20 \mathrm{~min}$, to remove cell debris. Finally, pre-cleaned secretome was concentrated to $\sim 1.5 \mathrm{~mL}$ using Centriprep YM-3 centrifugal units (Millipore, Bedford, MA, USA).

\section{Platelet rich plasma (PRP) Preparation}

Animals were anesthetized with $100 \mathrm{mg} / \mathrm{kg}$ ketamine10\% (Alfasan, Netherlands) and $10 \mathrm{mg} / \mathrm{kg}$ xylazine $2 \%$ (Alfasan, Netherlands). Then, venous blood was drawn from the heart, poured in a citrated dextrose tube and centrifuged at $1240 \mathrm{rpm}$ for $2 \mathrm{~min}$. The Supernatant layer, plasma fraction, composed of three distinct layers as follow: the upper layer was platelet-poor plasma (PPP), the middle layer was plasma average platelet (PAP) and the lower was platelet-rich plasma (PRP). The two top layers (PPP and PAP) were discarded by pipette. The third layer (PRP) was carefully separated by pipette and re-centrifuged at $1240 \mathrm{rpm}$ for $5 \mathrm{~min}$. Eventually, the plasma layer (upper layer) was separated and the PRP layer (second layer) was filtered to discard the leukocytes and then preserved for intra articular injection.

\section{Animal housing}

Thirty six adult male Sprague Dawley rats ( $200 \pm 20 \mathrm{~g}$ body weight) were used in the current research. All animals were kept in standard cages with $55 \pm 5 \%$ relative humidity, a 12-h light/dark cycle, and $22 \pm 2{ }^{\circ} \mathrm{C}$ temperature. All animals had free access to water and food ad libitum. The rats were randomly divided into 6 groups $(n=6)$. This study followed the internationally accredited guidelines with ethical approval from the Institutional Animal Care and Use Committee of Transgenic Research Center of Shiraz University of Medical Sciences (Shiraz, Iran). The registration number was 97-01-67-19128.

\section{Osteoarthritis induction}

General anesthesia was induced in rats by $100 \mathrm{mg} / \mathrm{kg}$ ketamine $10 \%$ (Alfasan, Netherlands) and 10 $\mathrm{mg} / \mathrm{kg}$ xylazine $2 \%$ (Alfasan, Netherlands), and then the rat knee was shaved. Four mg collagenase II (clostridium histolithicum; SigmaAldrich, St. Louis, MO, USA) dissolved in sterile PBS, and afterward was injected in the knee intra- articular space. After 3 days, injection was repeated.

\section{Animal grouping}

Two weeks after the second injection of collagenase II in which caused to induction of OA, animals were grouped as follow:

OA group: did not receive any treatment.

Positive group: taken an intra-articular Hyalgan injection ( $0.1 \mathrm{cc}$ with a concentration of $20 \mathrm{mg}$ manufactured by Fidia Italy). 
Treatment 1: taken an intra-articular injection of SMMSCs $\left(5 \times 10^{6}\right)$

Treatment 2: taken an intra-articular injection of SMMSCs $\left(5 \times 10^{6}\right)+$ secretome $(50 \mu \mathrm{l})$

Treatment 3: taken an intra-articular injection of SMMSCs $\left(5 \times 10^{6}\right)+$ PRP $(50 \mu \mathrm{l})$

Treatment 4: taken a combination of an intra-articular injection of SMMSCs $\left(5 \times 10^{6}\right)+$ secretome $(50 \mu l)$ + PRP $(50 \mu \mathrm{l})$

\section{Radiography evaluation}

Three months after onset of treatment, rats were sacrificed in a $\mathrm{CO} 2$ chamber, and thereupon radiography was taken from the lateral aspect of knee joint using radiographic equipment (AxiomMultix $\mathrm{M}$

Radiographic Unit, SiemensTM, Germany). Osteoarthritis in terms of presence of osteophytes, joint space narrowing, and subchondral sclerosis was assessed by a blinded radiologist.

\section{Histopathological study}

After sacrificing, the knee joints were harvested and fixed with $10 \%$ formalin solution for $48 \mathrm{hr}$. Afterward, samples were dehydrated with ethanol, embedded in paraffin, and cut into $4 \mu \mathrm{m}$ sections before staining. Hematoxylin and eosin (H\&E; Leica, Germany), Masson's trichrome (Merck, Germany), Toluidine blue (Merck, Germany), safranin O (Merck, Germany) and alcian blue (Merck, Germany) stains were used to evaluate cartilage structure, collagen formation, glycosaminoglycans [Molecular Weight ( $305 \mathrm{~g} / \mathrm{moles})$ ], sulfated glycosaminoglycans of proteoglycans [Molecular Weight (350 g/moles)], and glycosaminoglycans [Molecular Weight $(1341 \mathrm{~g} / \mathrm{moles})]$, respectively.

\section{Immunohistochemistry (IHC)}

IHC was performed using paraffin embedded tissue sections. Then, to do antigen retrieval technique heat induced epitope retrieval in citrate and Tris EDTA buffers was applied. Next, the samples were incubated in distilled water containing $3 \%(\mathrm{vol} / \mathrm{vol}) \mathrm{H} 2 \mathrm{O} 2$ for 10 min to block the endogenous enzymes. After washing, PBS containing goat serum for 60 minutes at room temperature was used to block the nonspecific binding sites. Thereafter, the samples were incubated with primary antibodies against SRYrelated protein 9 (SOX9; 1:250 dilution), matrix metalloproteinases 3(Mmp3; 1:100 dilution), and collagen type II (Col2) (1/200) (all from Abcam, UK) for $1 \mathrm{~h}$ at room temperature. Afterwards, the samples were incubated in polymer-HRP for $30 \mathrm{~min}$ and then diaminobenzidine was added as chromogen

\section{Statistical analysis}

All the experiments were performed in triplicate and analyzed using GraphPad Prism 5 software (GraphPad, La Jolla, CA). A value of $p<.05$ was considered as statistically significant.

\section{Results}


Figure 1a shows the spindle-shaped morphology of SMMSCs at passage 3 that acquired from the synovial membrane of male Sprague Dawley rat. Oil red-O staining and alizarin red staining are used to demonstrate the differentiation capacities of SMMSCs to adipocyte and osteocyte, respectively. Oil Red-O staining showed of lipid-rich vacuoles after 2 weeks (Fig. 1b). In addition, Alizarin Red staining revealed the formation of mineralized nodules after 2 weeks (Fig. 1c).

To characterize the phenotypes of SMMSCs, flow cytometry was executed to evaluate the surface markers of MSCs. Cells were labeled with FITC, perCP, and PE-conjugated antibodies and investigated by flow cytometry. Cells were stained with CD34, CD 45 (hematopoietic cell markers) and CD44, CD 90 (mesenchymal stem-cell markers). Based on our findings, the SMMSCs were negative for cell markers CD34, and CD45, while positive for MSC marker CD44 and CD90 (Fig. 1d-g).

\section{Histological assessments}

As revealed by the $\mathrm{H}$ and $\mathrm{E}$ staining, treatment groups ( $\mathrm{c} 1, \mathrm{~d} 1$, $\mathrm{e} 1$ and $\mathrm{f} 1$ ) had better articular cartilage surface continuity and integrity. More cell distribution was seen in, treatment 4 group ( $f 1)$, also, treatment 1, 2 and 3 groups (c1, d1 and e1) showed better cell distribution compared to OA (a1) and Hyalgan (b1) groups. In addition, treatment groups (c1, d1, e1 and f1) demonstrated better cartilage mineralization compared to OA (a1) and Hyalgan (b1) groups. Moreover, better subchondral bone was seen in treatment groups (c1, d1, and e1) especially in treatment 4 (f1). Besides, more cell population was observed in treatment groups (c1, d1, e1 and f1) especially in treatment 2 and 4 (d1 and f1). A regular and intact subchondral bone with normal interstitial space and lamellar orientations is seen in treatment groups (c1, d1 and f1) whereas treatment 3 (e1), OA (a1) and Hyalgan (b1) groups showed destruction of trabecular bone.

Those groups were received treatment groups (c2, d2, e2 and f2) exhibited more collagen type $\nabla$ using Masson's trichrome staining. A potent orientation of collagen is seen at superficial layer of treatment 4 (f2), and deep layer of treatment 2 (d2). In addition, those groups receiving stem cells demonstrated regular surfaces, while loss of superficial cell layers and surface irregularities are observed in OA (a2) and Hyalgan (b2) groups. Articular cartilage fraction and roughness were observed in some parts of e and $f$ groups.

Following toluidine blue staining, better extracellular matrix and orientation of chondrocytes were observed in those animals administrated by treatment groups (c3, d3 and f3). The cartilage layer in treatment groups (c3, d3 and f3) was heavily stained in comparision with OA (a3).

Based on safranin $0 /$ alcian blue staining, similar sulfated glycosaminoglycans of proteoglycan contents (red/orange) were observed in treatment groups (c1, e1 and f1) as the best findings. Likewise, treatment 2 (d1) and Hyalgan (b1) groups were the same in GAGs contents. Generally, all groups showed better results compared to OA group. 
Based on IHC, better expression of Col2 was observed in treatment groups $\mathrm{c} 2$ and f2. Besides, Similar Col2-positive cells was seen in treatment 2 (d2) and Hyalgan (b2) groups.

Lower expression of MMP3, as a proteolytic enzyme which is well known in the development and spread of inflammatory diseases such as osteoarthritis, was especially observed in treatment group 4 using IHC staining (Fig. 4). However, the level of MMP3 in OA and Hyalgan groups was significantly increased. Treatment groups 1, 2 and 3 exhibited less expression of MMP3 compared with untreated groups (Fig. 4).

To survey the potential effects of SMMSCs, secretome and PRP on OA-induced knee articular cartilage, IHC staining for SOX9 was done. Results showed higher expression of SOX9 in treatment groups 2, 3, and 4 (Fig. 5). Likewise, SOX9 -positive cells were more observed in superficial layer of articular cartilage in treatment group 4 (Fig. 5).

Joint space narrowing, subchondral sclerosis and osteophyte were not observed in treatment group 4. In treatment 1 and 2 groups, findings were normal but osteophyte was seen in early stage. A light Joint space narrowing and osteophytes in early stage were observed in treatment 3 group, while Joint space was normal. Joint space narrowing, moderate subchondral sclerosis and osteophyte were detected in OA and Hyalgan groups, while osteophyte formation was more severe in OA group.

\section{Discussion}

Osteoarthritis is a progressive and debilitating joint disease that needs to be overcome causes damage to the articular cartilage and the formation of new bone tissue in the periphery and center of the joint. It is notable that cartilage tissue has no blood vessels and nerves whereby treatment has been difficult and complicated. In line with this, cell therapy strategy is one of the most promising methods for repairing cartilage tissue lesions. Herein, the Synovial membrane-derived MSCs received groups showed promising findings concretely in combination with PRP and secretome in which can give an outlook on future work. Up to now, the effect of MSCs, PRP and secretome have studied in separated previous researches, but the synergistic effects of them on rat KOA has been investigated there. Our observations based on this biological compound demonstrated better effects in repair of cartilage in a comparable manner to their single use.

MSCs have impressive effect on cartilage repair in terms of their self-renewal capacity, differentiation into different types of host tissue cells along with secretion of important factors for cartilage regeneration and better function [13]. In addition, MSCs secrete pro-angiogenic factor which lead to improve the vasculature of subchondral bone and mobilize the circulating whereby cartilage destruction is deduced, blood supply in cartilage promoted and KOA repairment enhanced [14]. Fundamentally, MSCs are proper choice for repair because of their important roles by the secretion of VEGF and TGF to stimulate angiogenesis, interleukins 8 and 17 to act against inflammation, interleukin 6 and TNF to induce immunity, FGF and insulin-like growth factor (IGF) as anti-apoptotic factors, and also interleukins 4 and 10 as anti-oxidative agents $[15,16]$. 
In the present research, combination of the Synovial membrane-derived MSCs with PRP and secretome

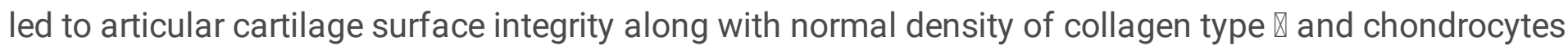
orientation. Likewise, glycosaminoglycans content and expression of matrix MMP-3 (metalloproteinases3) and SOX-9 (SRY-Box Transcription Factor-9) were improved. In parallel with our study, Ragab et al (2021) presented that the treatment of rat osteoarthritis using PRP results in reduction of destruction cartilage [17]. Meanwhile, Collagen II expression increment, cartilage surface loss and alleviation in the articular cartilage devastation were reported in those rats osteoarthritis which treated with PRP [18]. PRP as an autologous formulation from whole blood is enriched with some growth factors such as IGF, TGF- $\beta$ and PDGF which leads to stimulation of host cell proliferation and immigration. TGF- $\beta$ as an important growth factor binding to host cartilage stem cells in which stimulates them to secrete anti-inflammatory cytokines such as IL-4, IL-10 and IL-13 and also motivates these stem cells to differentiate into cartilage cells repairing the damaged articular cartilage as the chondroprotective effect $[19,20]$. Moreover, PRP as a safe remedy for protection of cartilage is stated that can relieve pain, ameliorate inflammation through decrement in TNF- $\alpha$ and IL-1 $\beta$ levels and delay in OA progression [21]. Also in accordance with the current results, smooth articular surface and normal joint space in radiography evaluation, articular cartilage hyper cellularity and returning of the articular cartilage to normal thickness based on H\&E staining and also moderate matrix staining according to Safranin $\mathrm{O}$ stain were observed in rats with induced-OA treating using PRP comprising diverse types of storage molecules for tissue healing and simplifying tissue regeneration [22]. The current observations are in accord with results from other researches in treatment of KOA.

Further, PRP intra-articular injection leads to chondrocytes increment. Moussa et al (2017) studied the effect of PRP on osteoarthritic chondrocytes resulting in augmentation of chondrocytes proliferation, collagen, and aggrecan while reduction in MMP-3 and apoptosis. Collagen creates a tensile strength in the tissue. Furthermore, aggrecan as one of the main proteoglycans leads to an increase in water absorption by chondrocytes, ameliorate articular cartilage movement and alleviate joint pain [23]. Consistent with our findings, Kusuma et al (2020) showed that treatment of KOA using Wharton's Jellyderived MSCs resulted in increase in collagen II expression and reduction of MMP3 as a matrix degradation mediator [24].

The MMPs is zinc- and calcium- dependent endopeptidases having the ability to proteolysis extracellular matrix (ECM) compounds and an important role in cartilage destruction by destroying cell surface proteins [25]. The MMPs family includes stromelysins, film sort, gelatinases, and cllagenases, thereby intensifies the inflammatory response cycle. As it was believed up to now, the lack of proteoglycans and weakening of the collagen meshwork make large ECM alteration in OA [26]. The MMPs as the basic enzymes causing ECM breakdown are produced by inflammatory cells such as macrophages, lymphocytes, neutrophils, and eosinophils, as well as by structural cells such as fibroblasts, epithelial, and endothelial cells. The MMP-3 as a member of stromelysins subfamily is small protease damaging the ECM constituents suchlike proteoglycan, and collagens results in obliteration of cartilage matrix deposition [27]. Moreover, MMP-3 is capable to agitate other MMPs to be active resulting in more demolition of cartilage structure. Compared to other groups, findings obtained from the IHC evaluation in 
the current research showed lower expression of MMP-3 in those group received Synovial membranederived MSCs in combination with PRP and secretome.

Studies have shown that during chondrogenesis, the Sox9 gene as a fundamental factor in developing cartilage is expressed simultaneously with the expression of the collagen II gene. In fact, the collagen II gene encoding is the target for the Sox 9 protein [28]. In the current research, Sox 9 expression along with preservation of collagen II were observed in Synovial membrane-derived MSCs in combination with PRP and secretome more than other groups. Zhi et al (2020) treated osteoarthritis in rat using bone marrowderived MSCs and stated that expression of aggrecan, collagen II, and SOX9 was considerably raised [29]. Also, Sox 9 markedly inhibits chondrocytes apoptosis and suppressed IL-1 $\beta$-induced growth prevention resulting in IL-1 $\beta$-stimulated aggrecan and collagen $\nabla$ reduction, and consequently notably decreased collagen-II degradation and the symptoms of OA. Furthermore, Luting et al (2021) demonstrated that PRP treatment for rabbit knee osteoarthritis resulted in stimulation the expression of Sox9. In addition, Sox 9 makes normal metabolism possible for cartilage cells in light of a lack of blood supply and hypoxic medium [30].

In 2019, Chen et al. reported that treatment of osteoarthritis in rat using secretome of human bone marrow-derived MSCs resulted in smooth and regular articular surface, and also preservation of GAGs and collagen II [3]. In addition, Khatab et al. (2018) stated pain reduction and protective efficacy on the evolvement of cartilage destruction after human bone marrow- derived MSCs injection in a mouse collagenase-induced OA model [31]. Clinically, increment of collagen II expression and reducing MMP-3 activity were reported in patients with $\mathrm{OA}$ treated using conditioned media from human adipose-tissuederived MSCs [32]. Furthermore, Tong et al. (2020) demonstrated that umbilical cord-derived MSCs treatment in rat-induced $O A$ deferred chondrocytes apoptosis, promoted cartilage superficial layer cells and preserved cartilage structure [33].

Based on the current experimental research, several limitations which need to be investigated in the future are as follow: First, evaluation using molecular tests to show the cellular functions and changes. Second, more in-depth investigations are essential in terms of angiogenesis, prevention of wear of the cartilage and inhibition of osteophyte formation. Last, it's necessary to perform this study in larger animals before clinical usage.

\section{Conclusions}

The current study revealed the important role of combination of the Synovial membrane-derived MSCs with PRP and secretome in the KOA treatment. In addition, betterment in KOA in terms of joint space, articular cartilage structure, sub chondral bone and ECM constituents were observed through inhibition the expression of MMP-3, and promotion the collagen II and Sox9 expression. In conclusion, injection the combination of the Synovial membrane-derived MSCs with PRP and secretome could be considered as a promising therapy for KOA patients. 


\section{Abbreviations}

SMMSCs: Synovial membrane-derived MSCs; PRP: platelet rich plasma; OA: Osteoarthritis; KOA: knee osteoarthritis; TGF- $\beta$ : transforming growth factor; PDGF: platelet-derived growth factor; PAP: plasma average platelet; DMEM: Dulbecco's modified Eagle's medium; MMP: matrix metalloproteinase; GAGs: Glycosaminoglycans; SOX: SRY-related high-mobility group (HMG) box; ECM: extracellular matrix; IL-1 $\beta$ : Interleukin 1 beta; IGF: Insulin-like growth factor; TNF: tumor necrosis factor; VEGF: Vascular endothelial growth factor; H\&E: hematoxylin and eosin.

\section{Declarations}

\section{Acknowledgements}

The authors would also like to thank animal lab technicians at Center of Comparative and Experimental Medicine Shiraz University of Medical Sciences, Shiraz, Iran.

\section{Authors' contributions}

All authors contributed to the study conception and design. NT, TTK, SSN and SD performed all the experiments, collected, and analyzed the data. SZ, MZ and OKH performed the animal surgery. SD wrote the manuscript. NT and TTK supervised the study. The authors read and approved the final article.

\section{Funding}

Not applicable.

\section{Availability of data and materials}

All data generated or analyzed during this experimental study are included in this published article.

\section{Ethics approval and consent to participate}

All experimental procedures and use of research animals in this study were approved by the Shiraz University of Medical Sciences ethics committee (97-01-67-19128).

\section{Consent for publication}

Not applicable.

\section{Competing interests}

The authors declare that they have no competing interests.

\section{References}


1. He L, He T, Xing J, Zhou Q, Fan L, Liu C, et al. Bone marrow mesenchymal stem cell-derived exosomes protect cartilage damage and relieve knee osteoarthritis pain in a rat model of osteoarthritis. Stem Cell Res Ther. 2020;11(1):1-15.

2. Bąkowski P, Kaszyński J, Wałecka J, Ciemniewska-Gorzela K, Bąkowska-Żywicka K, Piontek T. Autologous adipose tissue injection versus platelet-rich plasma (PRP) injection in the treatment of knee osteoarthritis: a randomized, controlled study-study protocol. BMC Musculoskelet Disord. 2020; $21: 1-8$.

3. Chen W, Sun Y, Gu X, Hao Y, Liu X, Lin J, et al. Conditioned medium of mesenchymal stem cells delays osteoarthritis progression in a rat model by protecting subchondral bone, maintaining matrix homeostasis, and enhancing autophagy. J Tissue Eng Regen Med. 2019;13(9):161828.

4. Wang Y, Zheng T, Song J, Gao W. A novel automatic Knee Osteoarthritis detection method based on vibroarthrographic signals. Biomed. Signal Process Control. 2021;68:1027-96.

5. Willinger ML, Heimroth J, Sodhi N, Garbarino LJ, Gold PA, Rasquinha V, et al. Management of Refractory Pain After Total Joint Replacement. Curr Pain Headache Rep. 2021;25(6):1-9.

6. Mohd Noor NA, Abdullah Nurul A, Ahmad Mohd Zain MR, Wan Nor Aduni WK, Azlan M. Extracellular Vesicles from Mesenchymal Stem Cells as Potential Treatments for Osteoarthritis. Cells. 2021;10(6):1287.

7. Sanjurjo-Rodriguez C, Altaie A, Mastbergen S, Baboolal T, Welting T, Lafeber F, et al. Gene Expression Signatures of Synovial Fluid Multipotent Stromal Cells in Advanced Knee Osteoarthritis and Following Knee Joint Distraction. Front Bioeng Biotechnol. 2020; 8.

8. Jeyaraman M, Muthu S, Gulati A, Jeyaraman N, GS P, Jain R. Mesenchymal Stem Cell-Derived Exosomes: A Potential Therapeutic Avenue in Knee Osteoarthritis. Cartilage. 2020;1947603520962567.

9. Mejia S. Identification of synovium secretome factor (s) for the prevention of osteoarthritis. Osteoarthr Cartil. 2021; 29:S127.

10. Russo R, Vassallo V, Stellavato A, Valletta M, Cimini D, Pedone PV, et al. Differential Secretome Profiling of Human Osteoarthritic Synoviocytes Treated with Biotechnological Unsulfated and Marine Sulfated Chondroitins. Int J Mol Sci. 2020;21(11):3746.

11. Filardo G, Previtali D, Napoli F, Candrian C, Zaffagnini S, Grassi A. PRP injections for the treatment of knee osteoarthritis: a meta-analysis of randomized controlled trials. Cartilage. 2020;1947603520931170.

12. Taniguchi Y, Yoshioka T, Kanamori A, Aoto K, Sugaya H, Yamazaki M: Intra-articular platelet-rich plasma (PRP) injections for treating knee pain associated with osteoarthritis of the knee in the Japanese population: a phase I and Ila clinical trial. Nagoya journal of medical science 2018, 80(1):39.

13. Xiao S, Liu W, Bi J, Liu S, Zhao H, Gong N, et al. Anti-inflammatory effect of hesperidin enhances chondrogenesis of human mesenchymal stem cells for cartilage tissue repair. J Inflamm. 2018;15(1):1-8. 
14. Fahy N, Alini M, Stoddart MJ. Mechanical stimulation of mesenchymal stem cells: Implications for cartilage tissue engineering. J Orthop Res. 2018;36(1):52-63.

15. Fortier LA, Barker JU, Strauss EJ, McCarrel TM, Cole BJ. The role of growth factors in cartilage repair. Clin Orthop Relat Res. 2011;469(10):2706-2715.

16. Hu Y, Chen X, Wang S, Jing Y, Su J. Subchondral bone microenvironment in osteoarthritis and pain. Bone Res. 2021;9(1):1-13.

17. Ragab G, Halfaya F, Ahmed O, El-Kheir A, Mahdi E, Ali T, et al. Platelet-rich plasma ameliorates monosodium iodoacetate-induced ankle osteoarthritis in the rat model via suppression of inflammation and oxidative stress. Evid Based Complement Alternat Med. 2021;2021.

18. Xin F, Wang H, Yuan F, Ding Y. Platelet-Rich Plasma Combined with Alendronate Reduces Pain and Inflammation in Induced Osteoarthritis in Rats by Inhibiting the Nuclear Factor-Kappa B Signaling Pathway. Biomed Res Int. 2020;2020.

19. Song H, Zhao J, Cheng J, Feng Z, Wang J, Momtazi-Borojeni AA, et al. Extracellular Vesicles in chondrogenesis and Cartilage regeneration. J Cell Mol Med. 2021;25(11):4883-92.

20. Kim YG, Choi J, Kim K. Mesenchymal Stem Cell-Derived Exosomes for Effective Cartilage Tissue Repair and Treatment of Osteoarthritis. Biotechnol J. 2020;15(12):2000082.

21. Xu Z, He Z, Shu L, Li X, Ma M, Ye C. Intra-articular platelet-rich plasma combined with hyaluronic acid injection for knee osteoarthritis is superior to platelet-rich plasma or hyaluronic acid alone in inhibiting inflammation and improving pain and function. Arthroscopy. 2021;37(3):903-15.

22. Ali A, Al-Attar A, Refat N, Gomaa M, Eldine Z, Samy A. Synergistic effect of nanocurcumin and platelet rich plasma on resolving experimentally induced osteoarthritis in albino rats. Adv Anim Vet Sci. 2021;9(1):26-34.

23. Moussa M, Lajeunesse D, Hilal G, El Atat O, Haykal G, Serhal R, et al. Platelet rich plasma (PRP) induces chondroprotection via increasing autophagy, anti-inflammatory markers, and decreasing apoptosis in human osteoarthritic cartilage. Exp Cell Res. 2017;352(1):146-156.

24. Kusuma HSW, Widowati W, Gunanegara RF, Juliandi B, Lister NE, Arumwardana S, et al. Effect of Conditioned Medium from IGF1-Induced Human Wharton's Jelly Mesenchymal Stem Cells (IGF1hWJMSCs-CM) on Osteoarthritis. Avicenna J Med Biotechnol. 2020;12(3):172.

25. Kosik-Bogacka DI, Lanocha-Arendarczyk N, Kot K, Zietek P, Karaczun M, Prokopowicz A, et al. Calcium, magnesium, zinc and lead concentrations in the structures forming knee joint in patients with osteoarthritis. J Trace Elem Med Biol. 2018;50:409-414.

26. Manka SW, Bihan D, Farndale RW. Structural studies of the MMP-3 interaction with triple-helical collagen introduce new roles for the enzyme in tissue remodelling. Sci Rep. 2019;9(1):1-14.

27. Wu Y, Lin Z, Yan Z, Wang Z, Fu X, Yu K. Sinomenine contributes to the inhibition of the inflammatory response and the improvement of osteoarthritis in mouse-cartilage cells by acting on the $\mathrm{Nrf} / \mathrm{HO}-1$ and NF-KB signaling pathways. Int Immunopharmacol. 2019;75:105715.

28. Zhang X, Wu S, Naccarato T, Prakash-Damani M, Chou Y, Chu C-Q, et al. Regeneration of hyaline-like cartilage in situ with SOX9 stimulation of bone marrow-derived mesenchymal stem cells. PloS one. 
2017;12(6):e0180138.

29. Zhi Z, Zhang C, Kang J, Wang Y, Liu J, Wu F, et al. The therapeutic effect of bone marrow-derived mesenchymal stem cells on osteoarthritis is improved by the activation of the KDM6A/SOX9 signaling pathway caused by exposure to hypoxia. J Cell Physiol. 2020; 235(10):7173-7182.

30. Luting Z, Qilin F, Haiyan T, Fangyuan X. The difference between autologous and allogeneic purified platelet-rich plasma in the treatment of rabbit knee osteoarthritis. Chinese J Tissue Eng Res. 2021;25(26):4123.

31. Khatab S, van Osch G, Kops N, Bastiaansen-Jenniskens Y, Bos K, Verhaar J, et al. Mesenchymal stem cell secretome reduces pain and prevents cartilage damage in a murine osteoarthritis model. Eur. Cells Mater. 2018;36:218-230.

32. Platas J, Guillén MI, del Caz MDP, Gomar F, Mirabet V, Alcaraz MJ. Conditioned media from adiposetissue-derived mesenchymal stem cells downregulate degradative mediators induced by interleukin$1 \beta$ in osteoarthritic chondrocytes. Mediators Inflamm. 2013; 2013.

33. Tong W, Zhang X, Zhang Q, Fang J, Liu Y, Shao Z, et al. Multiple umbilical cord-derived MSCs administrations attenuate rat osteoarthritis progression via preserving articular cartilage superficial layer cells and inhibiting synovitis. J OrthopTranslat. 2020;23:21-28.

\section{Figures}
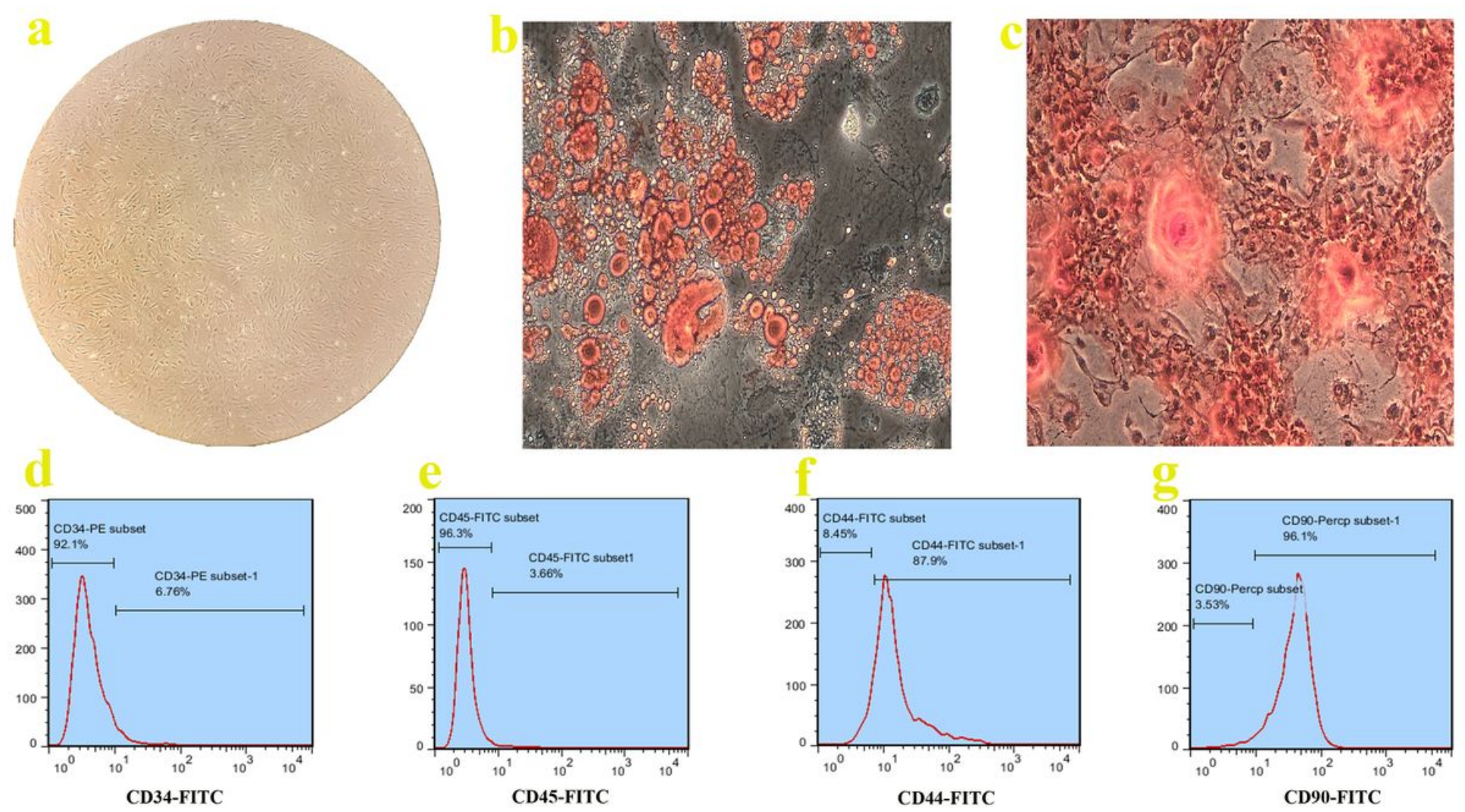

\section{Figure 1}


Morphology (a) (10x), differentiation capacity and cell surface markers of SMMSCs. For SMMSCs, adipogenic differentiation was shown by oil red staining (b), while osteogenic differentiation was demonstrated by calcium deposition in Alizarin red staining (c); $\times 10$ magnification. Flow cytometry study findings exhibited that SMMSCs were negative for hematopoietic markers CD34 (d) and CD45 (e) and also positive for MSC markers CD44 (f) and CD90 (g).

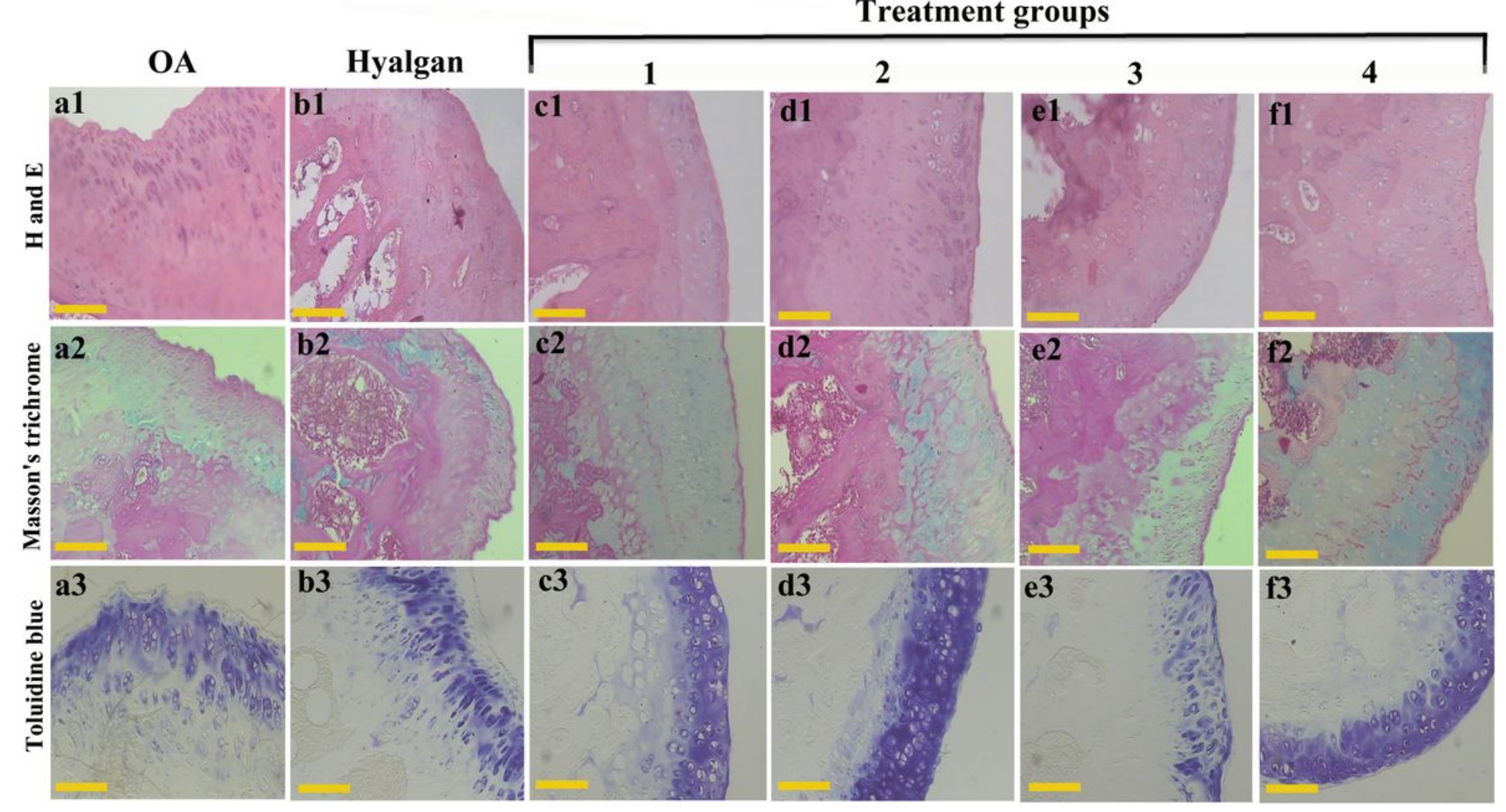

\section{Figure 2}

different stains for knee articular cartilage structure and constituents. $\mathrm{H}$ and $\mathrm{E}$ staining showed better articular cartilage surface continuity in treatment groups (c1, d1, e1 and f1). Masson's trichrome staining revealed more collagen type $\varangle(\mathrm{c} 2, \mathrm{~d} 2, \mathrm{e} 2$ and $\mathrm{f} 2)$. Toluidine blue staining exhibited better extracellular matrix preservation in treatment groups (c3, d3 and f3). (The yellow scale bar shows $200 \mu \mathrm{m}$ ). 


\section{Treatment groups}

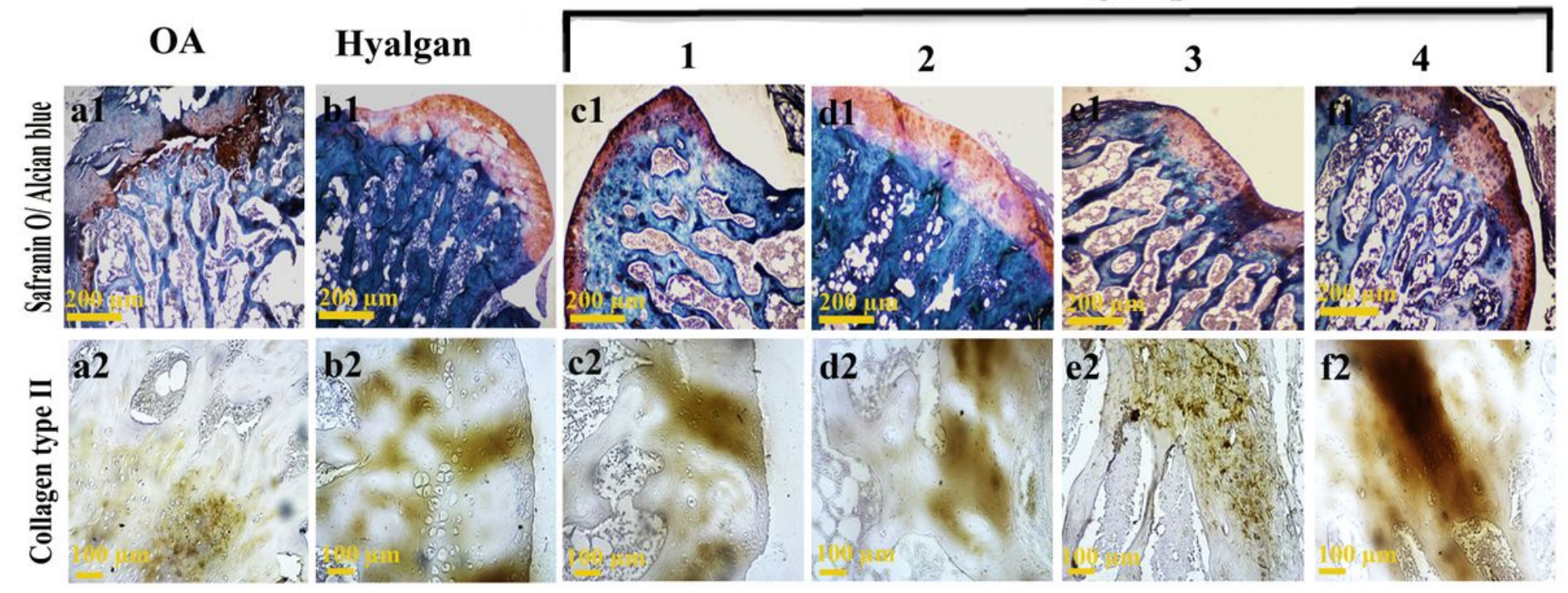

Figure 3

Special staining of knee articular cartilage. Safranin 0/ alcian blue staining showed more GAGs in treatment groups $\mathrm{c} 1, \mathrm{e} 1$ and $\mathrm{f} 1$. Immunohistochemistry for Col2 revealed better expression in treatment groups (c2 and f2).
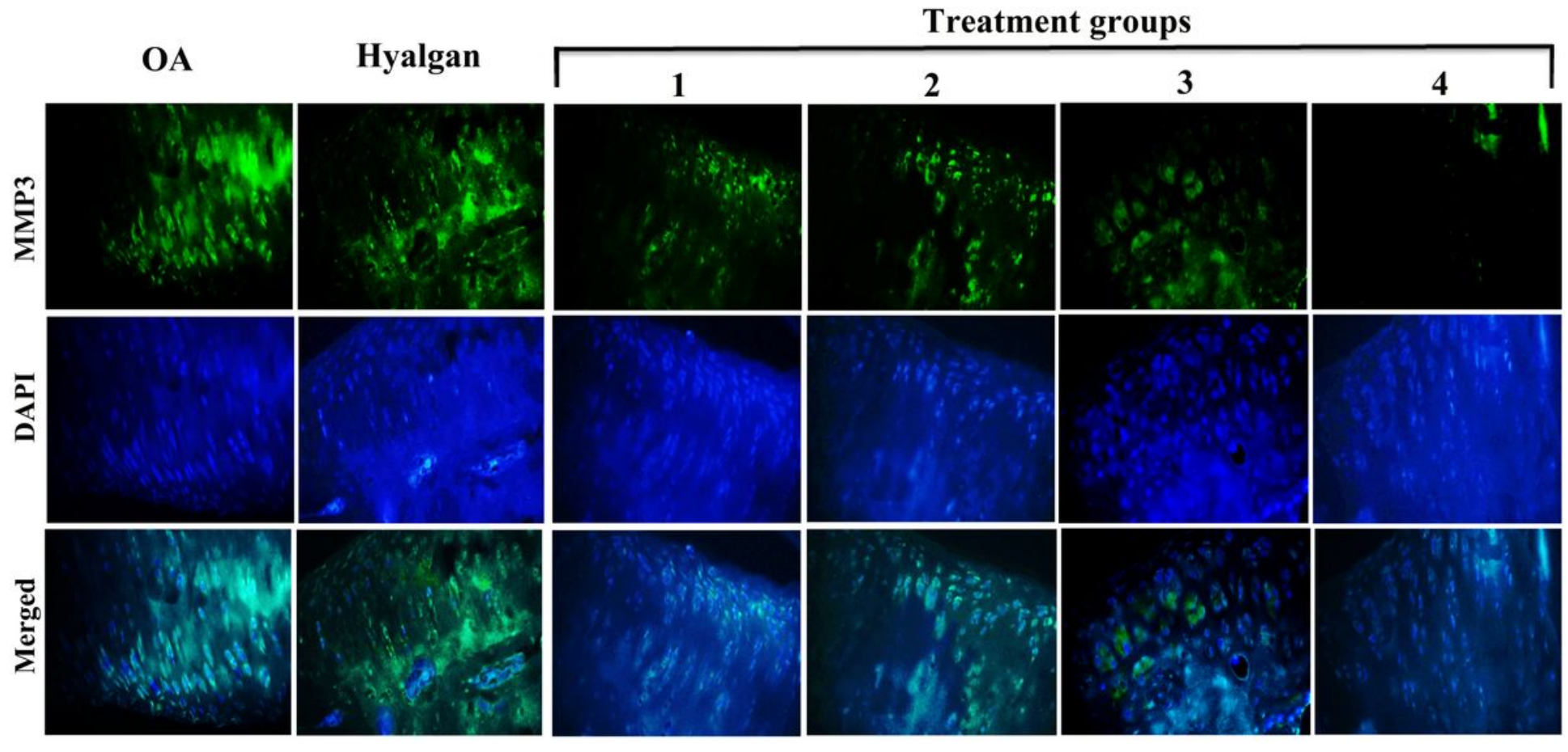

Figure 4

IHC evaluation of MMP3 in OA-induced knee articular cartilage. Compared with other groups, IHC revealed low expression of MMP3 in treatment group $4(10 \mathrm{x})$. 


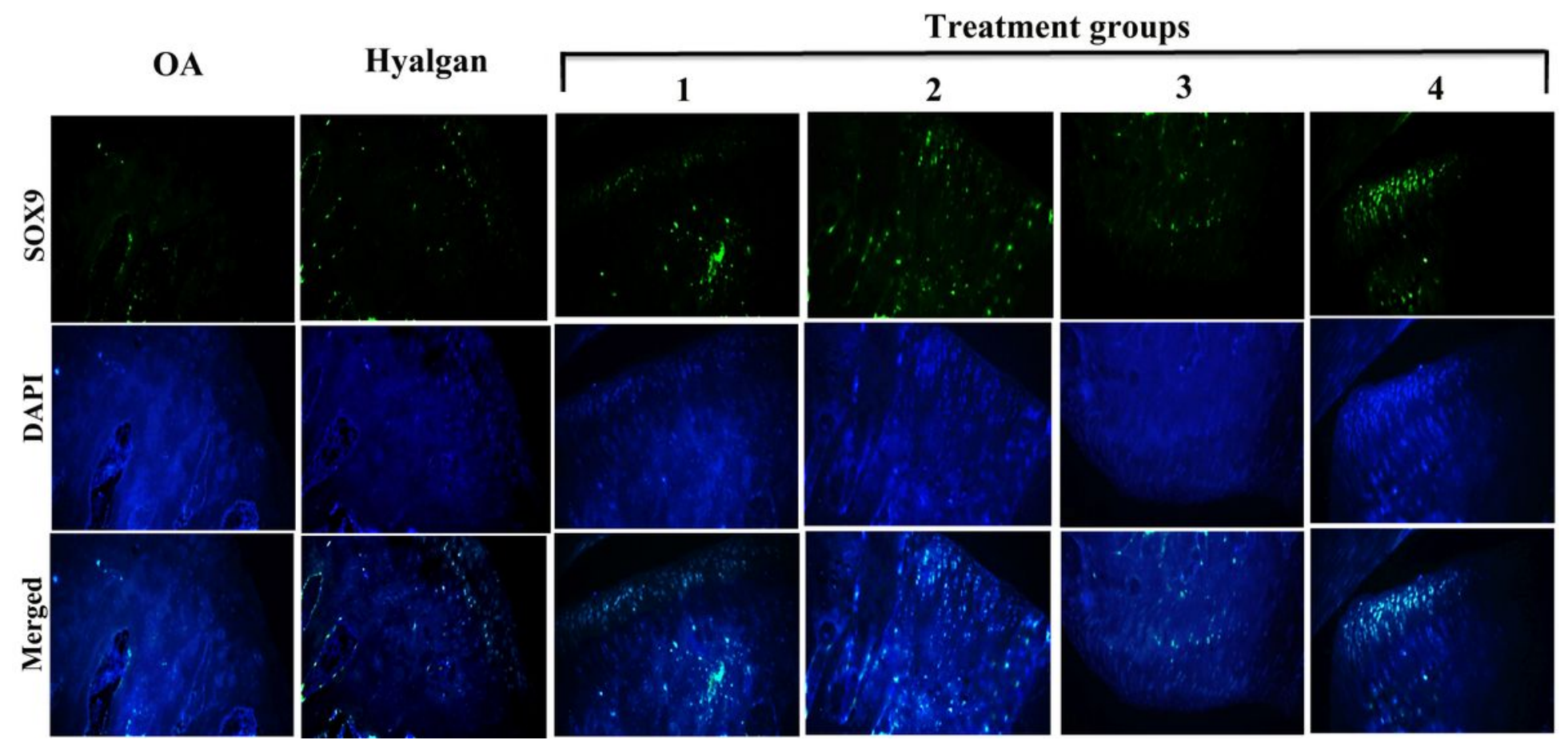

Figure 5

$\mathrm{IHC}$ evaluation of SOX9 in OA-induced knee articular cartilage. IHC revealed higher expression of SOX9 in treatment groups (2, 3 and 4$)(10 \mathrm{x})$.

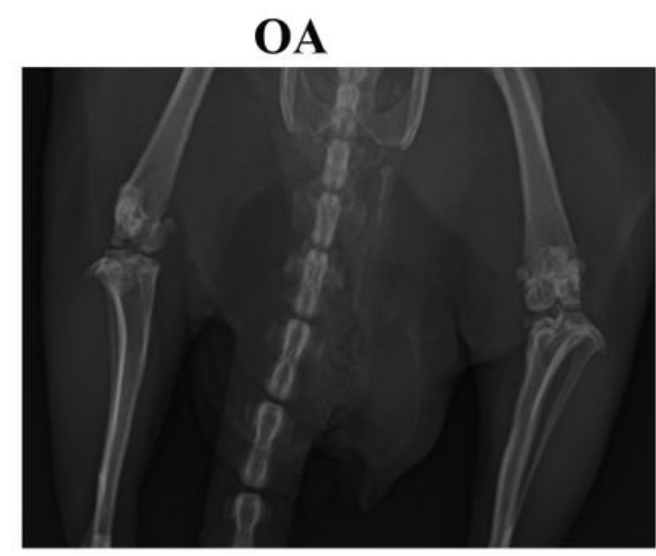

Treatment 2

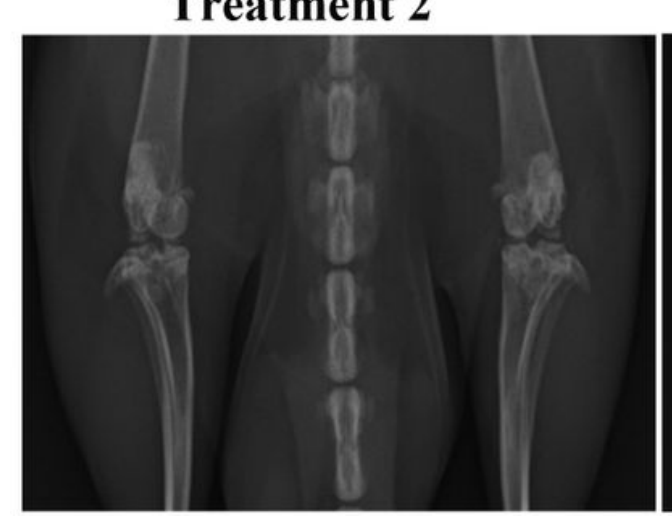

Hyalgan

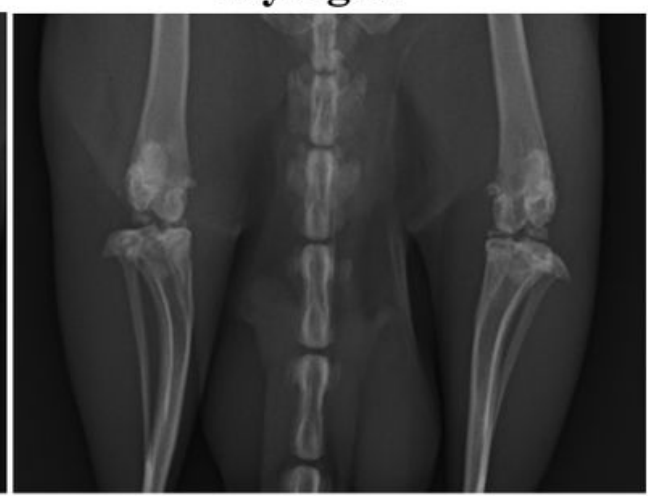

Treatment 3

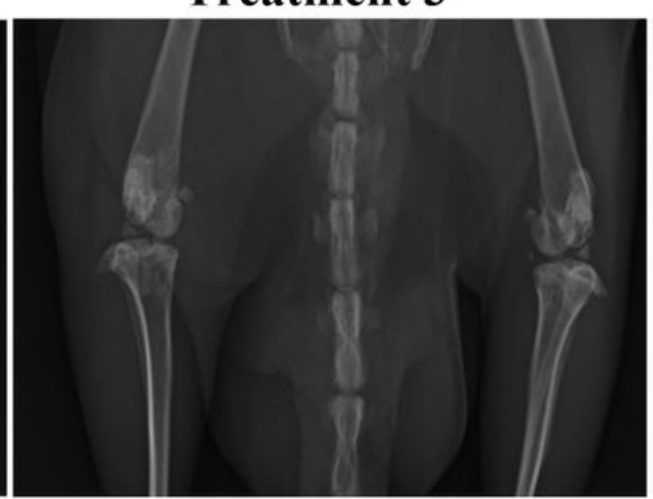

\section{Treatment 1}

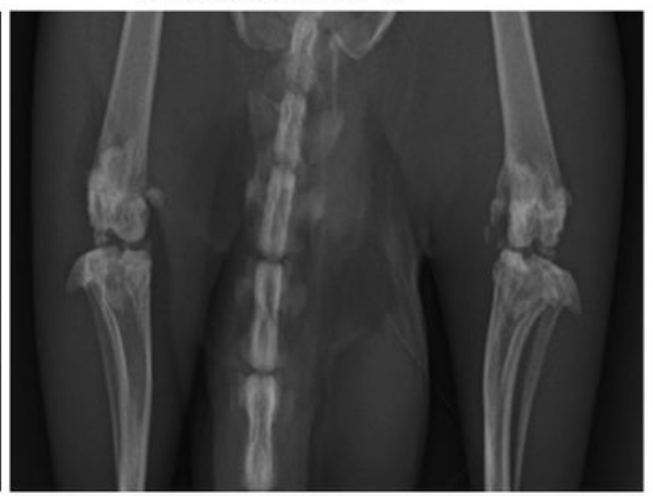

Treatment 4

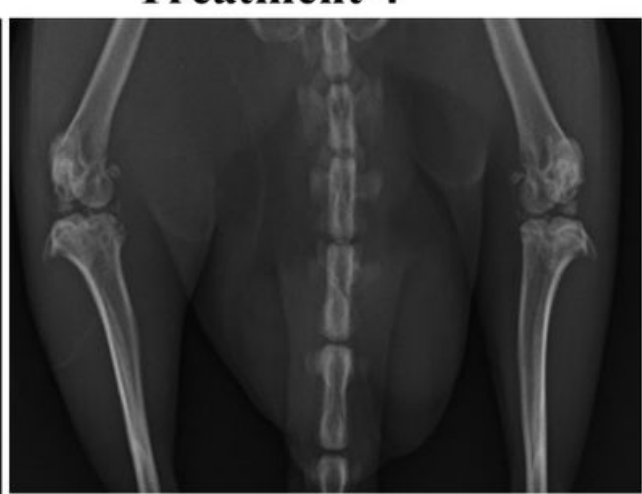

Figure 6 
Radiography Study. Joint space narrowing and osteophyte formation were observed in OA and Hyalgan groups. 